\title{
Matrix-induced autologous chondrocyte implantation for the treatment of chondral defects of the knees in Chinese patients
}

This article was published in the following Dove Press journal:

Drug Design, Development and Therapy

5 December 2014

Number of times this article has been viewed

\author{
Zhongwen Zhang' \\ Xin Zhong ${ }^{2}$ \\ Huiru $\mathrm{ji}^{1}$ \\ Zibin Tang' \\ Jianpeng Bai' \\ Minmin Yao' \\ Jianlei Hou' \\ Minghao Zheng ${ }^{3}$ \\ David J Wood ${ }^{3}$ \\ Jiazhi Sun ${ }^{4}$ \\ Shu-Feng Zhou ${ }^{4,5}$ \\ Aibing Liu ${ }^{6}$
}

'Department of Orthopedics, General Hospital of Chinese People's Armed Police Forces (CAPF), Beijing; '2Department of MRI Center, General Hospital of CAPF Beijing, People's Republic of China;

${ }^{3}$ Center for Orthopedic Research, School of Surgery and Pathology, University of

Western Australia, Perth, Western Australia,

Australia; ${ }^{4}$ Department of Pharmaceutical

Sciences, College of Pharmacy, University

of South Florida, Tampa, FL, USA; ${ }^{5}$ Guizhou

Provincial Key Laboratory for Regenerative

Medicine, Stem Cell and Tissue Engineering

Research Center and Sino-US Joint

Laboratory for Medical Sciences, Guiyang

Medical University, Guiyang, Guizhou;

${ }^{6}$ Medical Research Center, General Hospital

of Chinese People's Armed Police Forces

(CAPF), Beijing, People's Republic of China

Correspondence: Aibing Liu

Medical Research Center, General

Hospital of Chinese People's Armed

Police Forces (CAPF), 69 Yongding Road,

Beijing, People's Republic of China

Tel +86 1057975922

Fax +86 I0 68639576

Email doctor_liuaibing@aliyun.com

Shu-Feng Zhou

Department of Pharmaceutical Sciences,

College of Pharmacy, University of South

Florida, 1290I Bruce B Downs Blvd,

MDC 30, Tampa, FL, USA

Tel + I 8139746276

Fax + I 8I39059885

Email szhou@health.usf.edu
Abstract: Articular cartilage injury is the most common type of damage seen in clinical orthopedic practice. The matrix-induced autologous chondrocyte implant (MACI) was developed to repair articular cartilage with an advance on the autologous chondrocyte implant procedure. This study aimed to evaluate whether MACI is a safe and efficacious cartilage repair treatment for patients with knee cartilage lesions. The primary outcomes were the Knee Injury and Osteoarthritis Outcome Score (KOOS) domains and magnetic resonance imaging (MRI) results, compared between baseline and postoperative months 3, 6, 12, and 24. A total of 15 patients (20 knees), with an average age of 33.9 years, had a mean defect size of $4.01 \mathrm{~cm}^{2}$. By 6-month follow-up, KOOS results demonstrated significant improvements in symptoms and knee-related quality of life. MRI showed significant improvements in four individual graft scoring parameters at 24 months postoperatively. At 24 months, $90 \%$ of MACI grafts had filled completely and 10\% had good-to-excellent filling of the chondral defect. Most (95\%) of the MACI grafts were isointense and 5\% were slightly hyperintense. Histologic evaluation at 15 and 24 months showed predominantly hyaline cartilage in newly generated tissue. There were no postoperative complications in any patients and no adverse events related to the MACI operation. This 2-year study has confirmed that MACI is safe and effective with the advantages of a simple technique and significant clinical improvements. Further functional and mechanistic studies with longer follow-up are needed to validate the efficacy and safety of MACI in patients with articular cartilage injuries.

Keywords: articular cartilage lesion, Knee Injury and Osteoarthritis Outcome Score, KOOS, magnetic resonance imaging, MRI

\section{Introduction}

Articular cartilage injury is the most common type of damage seen in orthopedic practice. ${ }^{1-3}$ Since articular cartilage is avascular and aneural, this limits its ability to regenerate a biomechanically favorable hyaline-like repair tissue. This may invariably promote ongoing deterioration, with subsequent progression to early-onset osteoarthritis. ${ }^{4-6}$ While cartilage repair treatments such as lavage and debridement, microfracture, abrasion, mosaicplasty, marrow stimulation technique, and subchondral drilling, result in predominantly fibrous cartilage instead of hyaline cartilage with the clinical effects decreasing over time, ${ }^{2,7-12}$ autologous chondrocyte implantation (ACI) results in predominantly hyaline-like tissue regeneration. ${ }^{6,13-16}$ The ACI technique involves isolation of chondrocytes and proliferation in vitro to create a high-density chondrocyte suspension, which is injected to fill cartilage defects underneath a periosteal cover. Good clinical results have been documented with this technique. ${ }^{17-24}$ However, the large surgical incision, peripheral graft hypertrophy (25\% of patients) 
and calcification, and degeneration of sutured cartilage have compromised the efficacy of ACI. ${ }^{17-25}$

The matrix-induced autologous chondrocyte implant (MACI) was developed as the third and current generation ACI technique to repair articular cartilage, with advantages over the traditional ACI procedure. ${ }^{13,22,26,27}$ MACI has evolved based on the need to resolve complications associated with the use of periosteum, as well as the complexity and microtrauma of suturing the collagen cover and potential for cell leakage related to the aforementioned ACI techniques. ${ }^{27}$ With the MACI procedure, cultivated chondrocytes are seeded onto a type I/III collagen bilayer membrane, which is glued with fibrin sealant to the cartilaginous defect void after being trimmed to a suitable shape. ${ }^{13,22,26}$ Growth of a large number of chondrocytes seeded on the 3-dimensional membrane scaffold supports cell proliferation, promotes stable expression of their original phenotype, and enhances the chondrocyte-secreting matrix to increase the stiffness of the scaffold. ${ }^{13,22,26}$ The collagen membrane is characterized by good biocompatibility, suitable degradation time, and complete integration with the adjacent native cartilage. Use of a fibrin sealant also avoids a second injury caused by suturing, and the use of a nonautologous periosteum simplifies the operative procedure. With MACI, rather than suturing the defect cover, the cultured healthy chondrocytes are seeded directly and grow onto the collagen membrane in vitro and are then implanted into the defect and fixed in place with fibrin glue, which facilitates chondrocyte migration and proliferation. ${ }^{27}$ Using this technique, the implant does not have the same limitations encountered using the periosteal patch, and the surgery can be performed faster than both prior ACI predecessors and is less traumatic since only a smaller incision is needed to gain adequate defect exposure.

To date, there are many reports on the use of MACI in Western countries but none in Chinese patients. We hypothesized that MACI was a safe and effective approach for articular cartilage damage repair in Chinese patients. In this study, we evaluated 2-year clinical, radiologic, and histologic outcomes for patients with articular knee lesions who were treated with MACI, between July 2004 and July 2009, at the General Hospital of Chinese People's Armed Police Forces, which was the first and only hospital in the People's Republic of China to perform the MACI technique in patients.

\section{Materials and methods} Patient selection

Patients aged 14-60 years were enrolled and treated between July 2004 and July 2009, and were evaluated in accordance with the International Cartilage Repair Society (ICRS) grading guidelines and the Outerbridge criteria. ${ }^{28}$ The patients all had grade III/IV chondral defects of the patella or trochlear and failed nonsurgical therapies. All preoperative and postoperative treatments and evaluations including magnetic resonance imaging (MRI) were performed at the General Hospital of Chinese People's Armed Police Forces, Beijing, People's Republic of China. Exclusion criteria were body mass index $(\mathrm{BMI})>35$, bipolar lesions, ongoing inflammatory arthritis, $>3^{\circ}$ of varus/valgus malalignment, uncorrected congenital blood coagulation disorders, patients with a femoral epiphyseal growth plate that is not fully closed, or uncorrectable ligamentous deficiency.

\section{Ethics statement}

Ethics approval was obtained from the Clinical Ethics Committee of the General Hospital of Chinese People's Armed Police Forces, Beijing, People's Republic of China. All patients gave written informed consent and were treated according to the Helsinki Declaration and Chinese clinical study ethics laws and policies.

\section{Preoperative preparation}

All patients underwent radiographic examination of the knee after enrollment. Line of traction and Q angle were measured, and any findings of patellofemoral malalignment were noted for correction during the arthroscopic procedure or following MACI (ie, tibial tubercle ingression). Knee Injury and Osteoarthritis Outcome Score (KOOS), MRI, and laboratory examinations were conducted before surgery.

The cartilage defects were examined arthroscopically and concomitant pathologies such as patellar abnormalities were treated at this time. A $200 \mathrm{mg}$ sample of healthy cartilage was harvested from a non-weight-bearing area of the knee. The harvested cartilage was placed in a nutrient medium and sent for development of the matrix-induced autologous chondrocyte implant (MACI ${ }^{\circledR}$; Verigen, Perth, Australia), where the cells were passaged and seeded onto a type I/III collagen bilayer membrane (ACI-Maix; Matricel $\mathrm{GmbH}$, Herzogenrath, Germany) for implantation according to manufacturer instructions. The cell density was approximately one million cells per $\mathrm{cm}^{2}$.

\section{$\mathrm{MACl}$ surgical procedure}

The classic MACI technique was used as described previously. ${ }^{21,29}$ With the involved knee flexed at $60^{\circ}$, a parapatellar or midline incision of the knee was performed to expose the defect area. All damaged and loose cartilage down to the 
subchondral plate were removed from the defect, taking care to avoid bleeding. A foil template that reflected the geometry of the chondral defect was used to appropriately shape the MACI membrane, which was pressed into the defect and secured with a thin layer of fibrin glue (Tisseel; Baxter Healthcare, IL, USA). Stability of the MACI membrane was tested with passive flexion-extension movements.

\section{Postoperative rehabilitation}

The involved knee was flexed passively to $10^{\circ}$ and elevated at $15^{\circ}$ within 24 hours postoperatively. Activity was permitted in the involved knee (within $15^{\circ}$ ) protected by a brace and with no weight-bearing for the first week. The patient was discharged after 2-3 weeks provided that no abnormalities were found. Functional activities were allowed according to a rehabilitation program that progressively increased loading. Continuous passive motion was started at week 2 and the movement range was gradually increased to $60^{\circ}$ within 6 weeks; at the same time, isometric contraction exercises were permitted. After 6 weeks, the brace was eliminated and the patient was permitted to walk with crutches, followed by progressive increments of loading. Further activity guidelines and advice were provided until 12 months after surgery. After 6 months, circular motion and jogging were started; heavy exercise such as soccer was permitted after 12 months.

\section{Postoperative clinical and MRI evaluations}

The KOOS scoring system ${ }^{30,31}$ was used to evaluate pain, symptoms, activities of daily living, sports and recreation function, and knee-related quality of life (QoL) in patients at 1 week preoperatively and at 3, 6, 12, and 24 months postoperatively.

MRI examinations were performed preoperatively and at 3, 6, 12, and 24 months after surgery, and the results were evaluated by two experienced radiologists, both of whom were blinded to the data. MRIs were performed on a 3.0T MR unit (TrioTim; Siemens, Erlangen, Germany) or a 1.5T MR unit (Symphony; Siemens), and these following sequences were performed with a flexible surface knee coil: 1) sagittal SE T1WI; 2) sagittal FSE PDWI with fat-suppression (-FS); 3) coronal FSE PDWI-FS; 4) axial FSE T2WI-FS; and 5) sagittal threedimensional vibe T1WI-FS. A previously described system ${ }^{32}$ was used to evaluate several parameters: 1) degree of defect repair and filling of the $\operatorname{defect}(20=$ complete, $15=$ hypertrophy, $10=$ incomplete in $<50 \%$ of the adjacent cartilage, $5=$ incomplete in $>50 \%$ of the adjacent cartilage, and $0=$ subchondral bone exposed); 2) integration to the border zone ( $15=$ complete, $10=$ demarcating border visible, $5=$ defect visible for $<50 \%$ of the length of the repair tissue, and $0=$ defect visible for $>50 \%$ of the length of the repair tissue); 3) signal intensity of the repair tissue $(15=$ isointense, $10=$ slightly hyperintense, $5=$ moderately hyperintense, and $0=$ markedly hyperintense); and 4) condition of the subchondral bone ( $5=$ intact, $0=$ edema, granulation tissue, cysts, and sclerosis).

Three patients underwent arthroscopic examinations post-MACI treatment: one patient at 6 and 15 months, one at 8 months (to determine if MACI treatment would be worthwhile for the other knee), and one at 24 months (due to a meniscal tear during exercise). One histologic evaluation was taken at 15 months postoperatively.

\section{Statistical analysis}

Demographic and baseline characteristics were tabulated using appropriate summary statistics. Quantitative variables were calculated with the mean \pm standard deviation, and qualitative and ordinal variables were calculated with frequency and percentage. Full analysis set was the major statistical method used for effective analysis. We used repeated-measures analysis of variance, and $P<0.05$ was considered significant. A paired Student's $t$-test and $\chi^{2}$ test or signed-rank test was used to compare baseline and posttreatment variables. Two-tailed tests and SAS software (version 9.13; SAS Institute Inc., Cary, $\mathrm{NC}$, USA) were used for statistical analyses.

\section{Results}

\section{Patient characteristics}

A total of 15 patients were enrolled and treated between 2004 and 2009, for a total of 20 treated knees, and were followed up to 24 months. Six patients had lesions from osteochondritis dissecans, five from osteoarthritis, two from chondromalacia patella, and two from trauma. In three patients, tibial tubercle ingression was conducted concomitantly with MACI to improve patellofemoral joint alignment and reinforce the long-term efficacy of MACI. No patients were lost to follow-up over 24 months.

Patients' and defects' characteristics are presented in Table 1. Patients were mostly male (73\%) with an average age of 33.9 years (range 14-57 years). The average number of defects was 2.5 per patient (range: $1-6$ ), the mean defect size was $4.0 \mathrm{~cm}^{2}$ (range $0.5-12 \mathrm{~cm}^{2}$ ), and the defects were classified as grade III or grade IV according to the Outerbridge criteria and ICRS. ${ }^{28}$ The defects' average surface area were: $4.9 \mathrm{~cm}^{2}$ per medial femoral condyle $(\mathrm{n}=13) ; 3.1 \mathrm{~cm}^{2}$ per lateral femoral condyle $(n=4) ; 2.6 \mathrm{~cm}^{2}$ per patella $(\mathrm{n}=15)$; and $6.8 \mathrm{~cm}^{2}$ per trochlea $(\mathrm{n}=5)$. The average resurfacing area per knee $(\mathrm{n}=20)$ was $7.4 \mathrm{~cm}^{2}$. 
Table I Patient demographics and defect characteristics

\begin{tabular}{|c|c|}
\hline Characteristic & Number $(n, \%)^{a}$ \\
\hline Number of patients & 15 \\
\hline Number of knees receiving $\mathrm{MACl}$ & 20 \\
\hline \multicolumn{2}{|l|}{ Sex } \\
\hline Male & II (73) \\
\hline Female & $4(26)$ \\
\hline \multicolumn{2}{|l|}{ Age (years) } \\
\hline Mean \pm SD & $33.9 \pm 13.5$ \\
\hline Range & $14-57$ \\
\hline \multicolumn{2}{|l|}{ Distribution } \\
\hline$<16$ & I (7) \\
\hline$\geq 16$ to $<35$ & $9(60)$ \\
\hline$\geq 35$ to $<45$ & $2(13)$ \\
\hline$\geq 45$ to $<55$ & I (7) \\
\hline$\geq 55$ & $2(13)$ \\
\hline Number of defects & 37 \\
\hline \multicolumn{2}{|l|}{ Defect location } \\
\hline Medial femoral condyle & $13(35)$ \\
\hline Lateral femoral condyle & $4(I I)$ \\
\hline Patella & $15(40)$ \\
\hline Femoral trochlea & $5(13)$ \\
\hline \multicolumn{2}{|l|}{ Defect size $\left(\mathrm{cm}^{2}\right)$} \\
\hline Mean \pm SD & $4.0 I \pm 2.68$ \\
\hline Range & $0.5-12$ \\
\hline \multicolumn{2}{|l|}{ Distribution } \\
\hline$\leq 1.0$ & I (3) \\
\hline $1.0-1.9$ & $9(24)$ \\
\hline $2.0-2.9$ & $3(8)$ \\
\hline $3.0-3.9$ & $6(16)$ \\
\hline $4.0-4.9$ & $6(16)$ \\
\hline $5.0-5.9$ & I (3) \\
\hline $6.0-12.0$ & II (29) \\
\hline
\end{tabular}

Note: a Data are presented as $\mathrm{n}(\%)$ unless otherwise specified.

Abbreviations: $\mathrm{MACl}$, matrix-induced autologous chondrocyte implantation; $\mathrm{SD}$, standard deviation.

\section{Postoperative evaluation}

KOOS results are presented in Table 2. Except for sports and recreation, the mean KOOS scores for all four domains were over 70 at 12 months and over 80 at 24 months postoperatively. Overall, patients experienced a sustained improvement in their KOOS scores up to 24 months postoperatively. All domains at all time points, except for at 3 months, were significantly better than at baseline (Table 2). Significant reductions in symptoms $(P<0.05)$ were observed compared with baseline as early as 3 months. Compared with 3 months, all five KOOS domains had improved significantly $(P<0.05)$ at 6 months; and the same pattern was observed at 12 months compared with 6 months $(P<0.05)$. All five domains had improved significantly at 24 months $(P<0.05)$ when compared with the 12-month time point, except for QoL, which was maintained.

Postoperative MRI scores from follow-up at 3, 6, 12, and 24 months for all 37 lesions are found in Table 3. Between 3 and 24 months, mean changes were observed as follows: degree of defect repair and filling of the defect increased from 11.84 to 18.03 , integration to the border zone increased from 8.95 to 13.29 , signal intensity of the repair tissue increased from 3.55 to 14.34 , and score of the subchondral bone increased from 0.79 to 3.03. All four parameters showed significant improvements by 12 months: the defect areas were filled completely and repair tissue was almost fully integrated with adjacent cartilage, generated repair tissue was isointense with the surrounding tissue, and subchondral bone was almost normal. After 24 months, $90 \%$ of MACI grafts had filled completely, whereas $10 \%$ had good-toexcellent filling of the chondral defect. Most (95\%) of the MACI grafts were isointense, whereas $5 \%$ were slightly hyperintense. Integration of the MACI graft to the border zone was complete in $88 \%$ of patients, with the other $12 \%$ having a visible demarcating border. The subchondral bone was found intact in $60 \%$ of patients.

Figure 1 shows the MRI examination of one patient preoperatively, and at 6 months postoperatively. Integration with the adjacent native cartilage was nearly complete at 6 months, and no abnormality was found in the subchondral bone and marrow.

Table 2 KOOS evaluations over 24 months $(n=20)$

\begin{tabular}{|c|c|c|c|c|c|}
\hline \multirow[t]{2}{*}{ KOOS score } & \multirow[t]{2}{*}{ Baseline } & \multicolumn{4}{|c|}{ Follow-up (months) } \\
\hline & & 3 & 6 & 12 & 24 \\
\hline Number & 20 & 20 & 20 & 20 & 20 \\
\hline Pain & $52.37 \pm 15.63$ & $56.90 \pm 11.47$ & $69.05 \pm 6.23^{b}$ & $75.90 \pm 7.40^{c}$ & $82.65 \pm 7.03^{d}$ \\
\hline Symptoms & $46.06 \pm 14.59$ & $54.77 \pm 11.65^{\mathrm{a}}$ & $63.05 \pm 7.59^{b}$ & $79.25 \pm 6.80^{c}$ & $86.45 \pm 7.96^{d}$ \\
\hline ADL & $50.46 \pm 14.47$ & $50.77 \pm 10.13$ & $61.75 \pm 9.46^{b}$ & $71.20 \pm 9.75^{c}$ & $82.45 \pm 7.98^{d}$ \\
\hline Sports and recreation function & $34.91 \pm 17.06$ & $36.4 I \pm 9.47$ & $48.5 \pm 7.59^{b}$ & $62.35 \pm 9.76^{c}$ & $70.60 \pm 7.44^{d}$ \\
\hline Knee-related QoL & $50.82 \pm 8.64$ & $50.99 \pm 11.32$ & $64.25 \pm 6.94^{b}$ & $70.1 \pm 8.97$ & $83.75 \pm 7.76$ \\
\hline
\end{tabular}

Notes: Data are presented as mean $\pm S D$. ${ }^{a} P<0.05$, month 3 vs baseline. ${ }^{b} P<0.05$, month 6 vs baseline and month $3 .{ }^{c} P<0.05$, month 12 vs baseline and month 6 . ${ }^{d P}<0.05$, month 24 vs baseline and month 12 .

Abbreviations: ADL, activity of daily living; KOOS, Knee Injury and Osteoarthritis Outcome Score; QoL, quality of life; SD, standard deviation; vs, versus. 
Table 3 Mean scores of the MRI examinations of cartilage repair areas $(n=37)$

\begin{tabular}{lllll}
\hline Variables, mean \pm SD & \multicolumn{3}{l}{ Follow-up (months) } & $\mathbf{1 2}$ \\
\cline { 2 - 5 } & $\mathbf{3}$ & $\mathbf{6}$ & $\mathbf{2 4}$ \\
\hline Degree of defect repair and filling of the defect (total score =20) & $11.84 \pm 2.44$ & $14.87 \pm 2.72^{\mathrm{a}}$ & $17.24 \pm 2.52^{\mathrm{a}, \mathrm{b}}$ & $18.03 \pm 2.48^{\mathrm{a}, \mathrm{b}}$ \\
Integration to the border zone (total score $=15)$ & $8.95 \pm 3.88$ & $11.58 \pm 3.5 I^{\mathrm{a}}$ & $12.89 \pm 2.50^{\mathrm{a}, \mathrm{b}}$ & $13.29 \pm 2.40^{\mathrm{a}, \mathrm{b}}$ \\
Signal intensity of the repair tissue by dual T2-FSE (total score =15) & $3.55 \pm 2.58$ & $8.42 \pm 3.10^{\mathrm{a}}$ & $11.45 \pm 2.30^{\mathrm{a}, \mathrm{b}}$ & $14.34 \pm\left. 1.7\right|^{\mathrm{a}, \mathrm{b}, \mathrm{c}}$ \\
Subchondral bone (total score =5) & $0.79 \pm 1.85$ & $1.98 \pm 2.48^{\mathrm{a}}$ & $2.37 \pm 2.53^{\mathrm{a}}$ & $3.03 \pm 2.48^{\mathrm{a}, \mathrm{b}}$ \\
\hline
\end{tabular}

Notes: Data are presented as mean \pm SD and tested by repeated-measures analysis of variance between time points. ${ }^{\text {a }} P<0.05$, vs month 3 . ${ }^{\text {b } P<0.05}$, vs month $6 .{ }^{c} P<0.05$, vs month 12.

Abbreviations: FSE, fast spin echo; MRI, magnetic resonance imaging; SD, standard deviation; vs, versus.

Arthroscopic examinations were also performed on this patient at baseline (Figure 2A), and at 6 (Figure 2B) and 15 months (data not shown) postoperatively. The primary defect was filled with new repair tissue that was completely integrated with adjacent cartilage at 6 months. Similar findings were observed at 24 months postoperatively in another patient's arthroscopic examination (Figure 2D; for baseline, see Figure 2C). The newly generated repair tissue had color, luster, and hardness comparable to those of the healthy articular cartilage.

Finally, the repair tissue was histologically examined in the patient shown in Figure 1 and Figure 2B at 15 months, as well as in the patient shown in Figure 2D at 24 months postoperatively. Alcian blue, and hematoxylin and eosin (HE) staining of the generated repair tissue at 15 months showed predominantly hyaline-like repair tissue within a mixture of hyaline and fibrous cartilage (Figure 3). Similar findings were observed at 24 months in the second patient's histological examination (data not shown).

\section{Clinical rehabilitation}

The average time before initiating small range of motion with the involved leg with a brace was 2.7 weeks, and the average time before starting to walk with crutches was 5.6 weeks.

\section{Complications and failure}

Among all patients who underwent MACI, one patient experienced failure. At 24 months postoperatively, failure occurred in a 57-year-old male patient with a BMI of $>30 \mathrm{~kg} / \mathrm{m}^{2}$ and osteoarthritis. His lesions were located on the left medial femoral condyle $\left(7.5 \mathrm{~cm}^{2}\right)$, left patella $\left(4 \mathrm{~cm}^{2}\right)$, both tibial plateau and femur condyle corresponding area $\left(2 \mathrm{~cm}^{2}\right)$, right medial femoral condyle $\left(4 \mathrm{~cm}^{2}\right)$, and right patella $\left(8 \mathrm{~cm}^{2}\right)$.
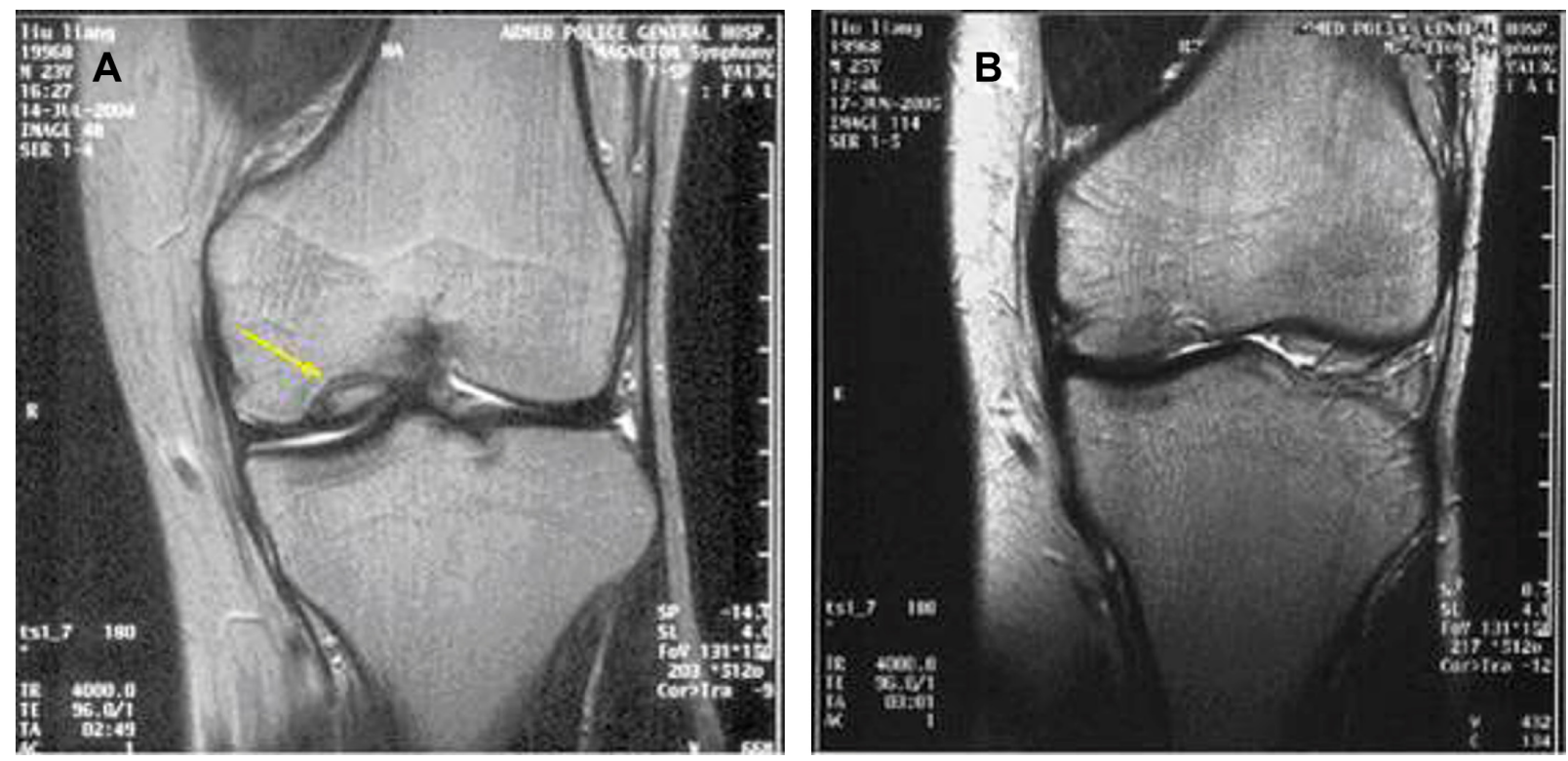

Figure I MRI of the knee joint before and after the $\mathrm{MACl}$ operation.

Notes: (A) The cartilage defect region before surgery (yellow arrow), and (B) complete filling of the defect area at 6 months after surgery.

Abbreviations: $\mathrm{MACl}$, matrix-induced autologous chondrocyte implantation; $\mathrm{MRI}$, magnetic resonance imaging. 

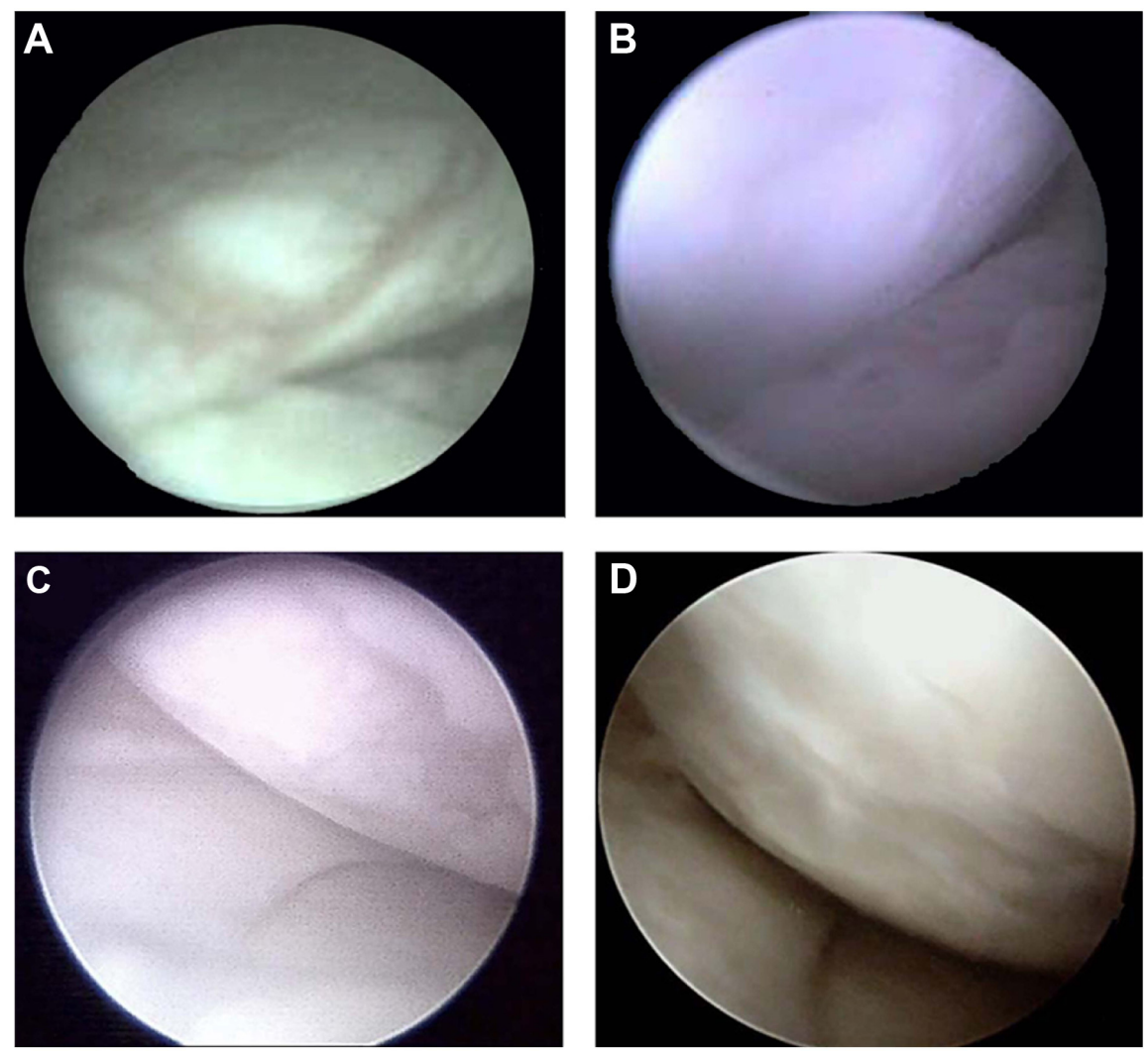

Figure 2 Arthroscopic images in two patients before operation and at 6 and 24 months after surgery.

Notes: (A) Preoperative image of the articular cartilage defect with a rough surface, (B) at 6 months after surgery, the defect was filled with new repair tissue that had a smooth surface and good integration, (C) the cartilage defect in another patient preoperatively, and (D) image at 24 months after surgery; the color, luster, and hardness of the newly generated repair tissue were comparable to those of healthy cartilage, and the repair tissue was integrated well with the surrounding cartilage.
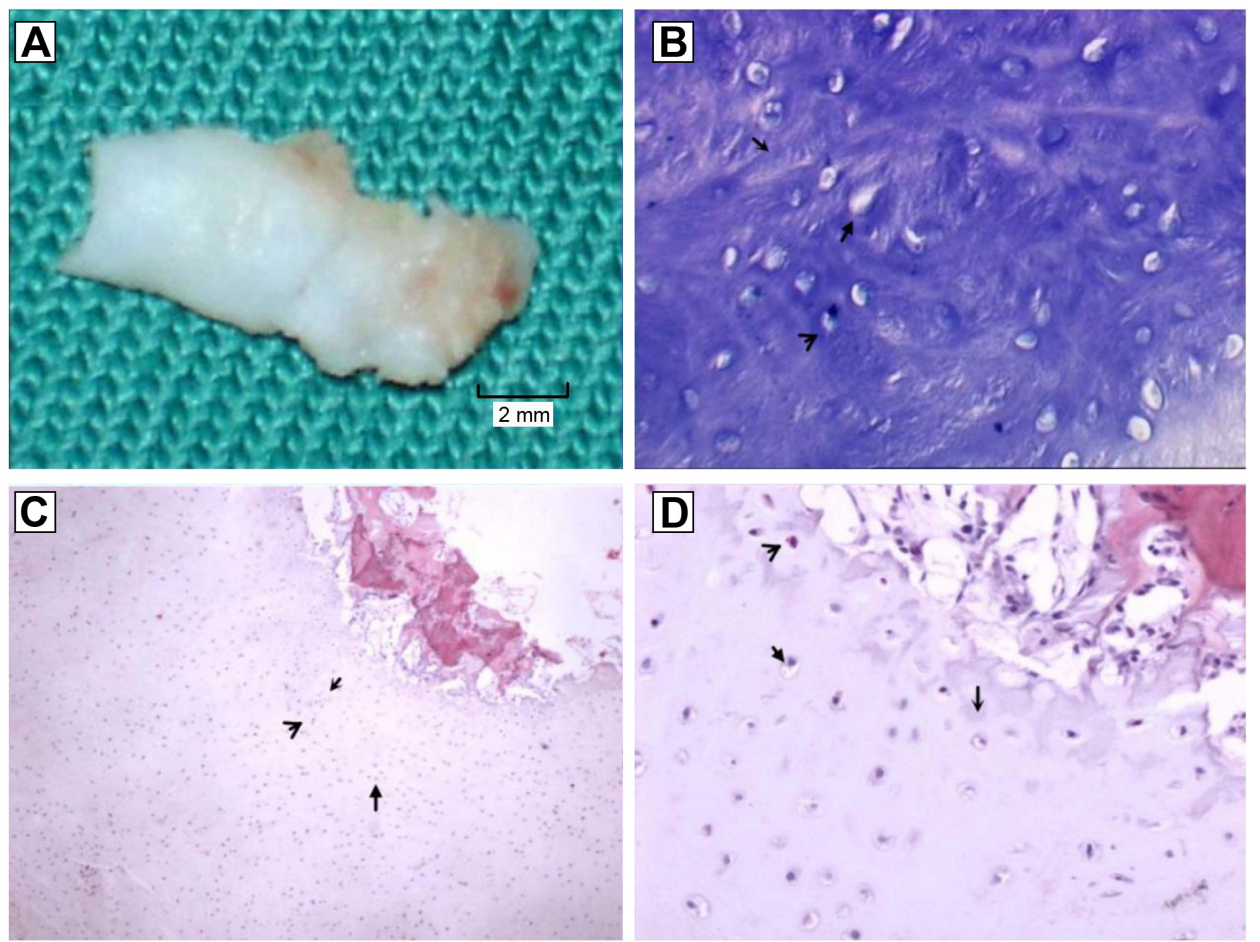

Figure 3 Histologic examination of the biopsies.

Notes: (A) Biopsy of the generated repairing tissue, (B) alcian blue stain $(\times 200),($ C) hematoxylin and eosin stain $(\times 40)$, and $($ D) hematoxylin and eosin stain $(\times 200)$ of repairing tissue at 15 months after surgery, which show the predominance of hyaline-like repair tissue. Hyaline cartilage, cartilage lacuna, and infantile chondrocytes are indicated by arrows. 
The patient had both knees treated with the MACI procedure with a concomitant osteotomy in the right knee; the tibial plateau lesions were not treated. Two years prior to the MACI treatment, the patient's left knee had been treated with microfracture. The patient had a total knee replacement on the right 5 years after treatment. It is noteworthy to mention that both KOOS and MRI scores over time still improved despite the failure of the MACI procedure.

Among all patients receiving the MACI procedure, no postoperative complications or adverse events such as infective arthritis, wound infection, joint swelling, joint stiffness, bone edema, hyperthermia, pyrexia, and implant site edema were reported. No graft delamination, graft complication, graft hypertrophy, graft loss, or cartilage injury was reported.

\section{Discussion}

Several critical factors may determine clinical outcomes and quality of repair tissue after MACI procedure in patients, including 1) successful chondrocyte culture and expansion, 2) technical proficiency of the surgeons who conduct the procedure, 3) patient cooperation and compliance in all aspects of the preoperative and postoperative programs and guideline offered by the surgeons, and 4) timely progression of weight-bearing and adjunct exercises and postoperative rehabilitation..$^{25,27}$ In this first report from the People's Republic of China, we confirmed our hypothesis that MACI implantation is a safe and efficacious cartilage repair treatment in patients. In our study, MACI implantation significantly reduced pain and symptoms and improved function and knee QoL in patients with lesions from trauma, osteoarthritis, and osteochondritis dissecans. In addition, good cartilage repair tissue was seen arthroscopically and histologically, as observed by mostly complete defect fill, almost complete integration with adjacent cartilage, and a high amount of hyaline-like repair tissue. Lastly, our MACI experience shows that the implantation procedure, although open, could be completed without complications or procedure-related adverse events.

All patients had sustained improvements in KOOS scores up to 24 months postoperatively for the domains of pain, symptoms, activities of daily living, sports and recreation function, and knee-related QoL $(P<0.05)$. At 24 months postoperatively, all mean KOOS scores (Table 2) were over 80 , except for the sports and recreation function, which was 70. This was likely related to psychological factors, as patients may not have been willing to resume activities for fear of a repeat injury. We suggest encouraging patients to do appropriate exercises and get early, active rehabilitation, as recommended by Ebert et al. ${ }^{33,34}$ Furthermore, early patient improvements were noted. Similar to our results, significant improvements from baseline were shown in all five KOOS domains at 24 months in a case series of young patients undergoing a combined high tibial osteotomy and MACI because of medial knee osteoarthritis, ${ }^{35}$ and in another case series of patients treated with MACI because of persistent pain associated with grade III or IV chondral lesions. ${ }^{32}$

At 3 months postoperatively, MRI for defect repair and filling showed integration of the border zone exceeding $50 \%$, signal intensity of the repair tissue that had markedly increased, and subchondral bone edema in the majority of repaired tissue. By 6 months postoperatively, MRI showed defect tissue repair and filling with integration to the border zone approaching $80 \%$; signal intensity of the repair tissue was moderately hyperintense, and subchondral bone edema approached $60 \%$. At 12 and 24 months postoperatively, the repair tissue was filled completely and almost fully integrated with adjacent cartilage, was isointense with the surrounding tissue, and the subchondral bone was almost normal. Overall, the recovery of the subchondral bone was slow. Similar MRI results demonstrating good repair tissue with good defect filling and integration at 24-30 months were reported in other studies. ${ }^{36-38}$ However, longer term studies have shown some deterioration in the repair tissue at 60 months. ${ }^{32,35,36}$

For those patients who underwent arthroscopic examination, the images showed that the primary defects were filled with new repair tissue and completely integrated with adjacent cartilage by 6 months postoperatively. Histologic examination of generative repair tissue at 15 and 24 months exhibited a predominance of hyaline-like tissue within a mixture of hyaline and fibrous tissue, indicating that the MACI procedure has the potential for long-term effectiveness. This also indicates the success of implantation of healthy chondrocytes, which proliferate and synthesize new collagens and other main matrix components.

In this study, we used porcine-derived type I/III collagen bilayer membrane ACI-Maix from Matricel $\mathrm{GmbH}$ for implantation of cultured healthy chondrocytes ex vivo. The biodegradable collagen matrix is considered to play an important role in cartilage repair after the MACI procedure, which supports the growth, migration, and proliferation of chondrocytes in the defected locations. ${ }^{25,27}$ The collagen membrane will be resorbed, a proteolytic process performed by cells in the vicinity of the defects over the months following implantation.

Many variables may influence the clinical outcomes of patients treated with MACI, including age, BMI, chondral defect size, location and mechanism of injury, joint degeneration at the time of surgery, ex vivo chondrocyte culture 
procedure, preoperative duration of symptoms, number of prior knee surgeries, and postoperative rehabilitation. ${ }^{26,39,40}$ The use of MACI is not recommended in patients over 65 years of age who have generalized degeneration of the cartilage or osteoarthritis. In this study, we only recruited patients aged 14-60 years. Age has demonstrated a significant negative impact on the clinical outcomes of MACI, implying that the gradient and intensity of postoperative rehabilitation may require a more conservative approach in an older patient.

BMI also has a significant negative impact on the clinical outcome of MACI.,41,42 For better clinical outcomes, BMI should be less than $30 \mathrm{~kg} / \mathrm{m}^{2}$, and it has been demonstrated that any reduction in body weight results in a 4-fold reduction in loads experienced at the knee during normal ambulation and daily activities. ${ }^{43}$ This highlights the importance of preoperative weight loss and postoperative weight maintenance, particularly for tibiofemoral weight-bearing grafts. Rehabilitation for patients with excess body weight may require a more conservative progression in the return to full weight-bearing gait. ${ }^{27}$

Chondral defect size has been shown to have a significant negative effect on the clinical outcomes of MACI. ${ }^{27}$ Some authors have suggested that an upper limit of $7.5 \mathrm{~cm}^{2}$ may exist and after this level, a poorer graft outcome may be observed. ${ }^{27,44}$ A recent 5-year follow-up study has demonstrated that factors such as graft size, duration of symptoms, preoperative Medical Outcomes Study 36-Item Short-Form Health Survey scores, and the postoperative course of weightbearing rehabilitation are important factors contributing to positive 5-year clinical and radiological outcomes and overall patient satisfaction after MACI ${ }^{44}$ In our study, the mean defect size was $4.0 \mathrm{~cm}^{2}$, ranging $0.5-12 \mathrm{~cm}^{2}$. We have noted that patients with defect sizes of $>7.5 \mathrm{~cm}^{2}$ also showed good clinical outcome after MACI. More studies are needed to explore the impact of chondral defect size on the clinical outcomes of MACI.

In general, the MACI procedure was safe based on the exposure of a large number of patients to MACI treatment in the knee. ${ }^{45,46}$ Complications of MACI may be related to the arthrotomy procedure, general complications related to surgical intervention, other knee pathology (such as ligamentous or meniscal pathology), or the biopsy procurement. Complications related to knee surgery in general may also include deep vein thrombosis and pulmonary embolism. In this study, except for one failure after 24 months in a patient with multiple lesions, none of the patients reported postoperative complications, and there were no adverse events related to MACI.
Following MACI, four stages of tissue/graft maturation have been described: $\left.{ }^{39} 1\right)$ graft implantation and chondrocyte attachment ( $0-6$ weeks): the type I/III collagen membrane with seeded chondrocytes is glued onto the subchondral bone using fibrin glue; 2) graft transition and chondrocyte proliferation (6-12 weeks): chondrocytes migrate from the membrane through the fibrin sealant to the subchondral bone and fill the defect with a soft repairing tissue; 3) graft and tissue remodeling (12-26 weeks): chondrocytes generate a matrix consisting of type II collagen, aggrecan (also known as cartilage-specific proteoglycan core protein or chondroitin sulfate proteoglycan 1), glycosaminoglycans, and other matrix proteins, and along with type II collagen, aggrecan forms a major structural component of the cartilage; and 4) graft maturation (6 months to 3 years): the chondrocytes and matrix reach full maturity and cartilage function recovers. During this stage, the graft is well-integrated with the adjacent native cartilage and underlying bone. Since the maturation process following MACI will last for up to 3 years, a proper follow-up program of the patients is important to ensure successful graft maturation.

MRI has demonstrated efficacy in the evaluation of tissue repair following MACI and the magnetic resonance observation of cartilage repair tissue (MOCART) classification has been the most common system utilized for cartilage repair, while a number of MRI scoring systems have been reported..$^{47,48}$ In our study, we observed a largely consistent improvement of the knee cartilages by both MRI and clinical evaluations. However, the MRI-based findings may be in conflict with the clinical assessments. ${ }^{47-49}$ Graft hypertrophy and signal intensity were the strongest MRI-based correlates with clinical outcomes after ACI, and others have found that the overall MRI score and graft infill correlated most often with clinical outcomes following a range of cartilage repair procedures. ${ }^{48}$ In a recent retrospective study with 83 patients receiving MACI surgery, Ebert et al ${ }^{42}$ did not find any correlations between graft infill, signal intensity, or the composite score with the KOOS domains.

On the other hand, the histological findings may not be in agreement with radiological and clinical observations in patients undergoing MACI. In one recent prospective multicentered study with 30 patients treated with MACI, the histological outcome was not significantly related either to the macroscopic appearance of the lesion or to the patient's functional status at the time of biopsy. ${ }^{26}$ Since the graft maturation after MACI will take up to 3 years, the healing and maturation process after MACI needs to be better understood at the molecular, histological, and clinical levels, and a longer 
follow-up is needed to better clarify the relationship between histology, radiology, and long-term clinical outcomes.

Contraindications for undertaking MACI include coexistent knee joint malalignment, ligamentous instability, and/or meniscal deficiency. ${ }^{27}$ Anterior cruciate ligament deficiency alters in vivo cartilage contact biomechanics by shifting the contact location to smaller regions of thinner cartilage and by increasing the magnitude of cartilage contact deformation. Adjunct surgical procedures may be carried out in combination with the MACI surgery. In this study, we excluded the patients with the above indications to avoid poor clinical outcomes.

Limitations of our study include the fact that this was a prospective case series, which lacked a control or comparative cohort and that it only consisted of 15 patients. However, none of the patients was lost to follow-up. In addition, the etiology of the lesions was several fold, such as osteochondritis dissecans, osteoarthritis, chondromalacia patella, and trauma, and the lesions were located in different areas. Such variation results in a heterogeneous population, and although results may vary depending on etiology or lesion location, the number of patients here was too small for any stratification analyses. Despite these variations, the overall population improved with MACI implantation.

The outcomes of our first clinical study of MACI implantation in the People's Republic of China have showed that it is a valid procedure to relieve patients' pain and improve function by generating new repair tissue treat lesions of various etiologies. Compared with conventional operations and ACI, the significant advantages of MACI include technical simplicity, minimal invasiveness, and significant improvements. Thus, the MACI technique is an effective choice for patients with pain, symptoms, and adversely affected function because of articular cartilage lesions. Further functional and mechanistic studies are needed to validate the efficacy and safety of MACI in patients with articular cartilage injuries.

\section{Acknowledgments}

The authors acknowledge Ms Kathleen Ohleth, $\mathrm{PhD}$ (Precise Publications LLC, Bedminster, NJ, USA) for editorial assistance in preparation for the manuscript, which was supported by Sanofi Biosurgery Inc., Cambridge, MA, USA (formerly Genzyme Biosurgery).

\section{Disclosure}

Sanofi Biosurgery (formerly Genzyme Biosurgery) provided support for editorial assistance in preparation for manuscript submission. The authors report no other conflicts of interest in this work.

\section{References}

1. Marcacci M, Filardo G, Kon E. Treatment of cartilage lesions: what works and why? Injury. 2013;44 Suppl 1:S11-S15.

2. Moran CJ, Pascual-Garrido C, Chubinskaya S, et al. Restoration of articular cartilage. J Bone Joint Surg Am. 2014;96(4):336-344.

3. Davis AM, MacKay C. Osteoarthritis year in review: outcome of rehabilitation. Osteoarthritis Cartilage. 2013;21(10):1414-1424.

4. Behery O, Siston RA, Harris JD, Flanigan DC. Treatment of cartilage defects of the knee: expanding on the existing algorithm. Clin J Sport Med. 2014;24(1):21-30.

5. Buckwalter JA, Hunziker EB. Orthopaedics. Healing of bones, cartilages, tendons, and ligaments: a new era. Lancet. 1996;348 Suppl 2:sII18.

6. Brittberg M, Lindahl A, Nilsson A, Ohlsson C, Isaksson O, Peterson L. Treatment of deep cartilage defects in the knee with autologous chondrocyte transplantation. N Engl J Med. 1994;331(14):889-895.

7. El Bitar YF, Lindner D, Jackson TJ, Domb BG. Joint-preserving surgical options for management of chondral injuries of the hip. J Am Acad Orthop Surg. 2014;22(1):46-56.

8. Haddad B, Konan S, Haddad FS. Debridement versus re-attachment of acetabular labral tears: a review of the literature and quantitative analysis. Bone Joint J. 2014;96-B(1):24-30.

9. Mithoefer K. Complex articular cartilage restoration. Sports Med Arthrosc. 2013;21(1):31-37.

10. McCormick F, Harris JD, Abrams GD, et al. Trends in the surgical treatment of articular cartilage lesions in the United States: an analysis of a large private-payer database over a period of 8 years. Arthroscopy. 2014;30(2):222-226.

11. Triche R, Mandelbaum BR. Overview of cartilage biology and new trends in cartilage stimulation. Foot Ankle Clin. 2013;18(1):1-12.

12. Goyal D, Keyhani S, Lee EH, Hui JH. Evidence-based status of microfracture technique: a systematic review of level I and II studies. Arthroscopy. 2013;29(9):1579-1588.

13. Roelofs AJ, Rocke JP, De Bari C. Cell-based approaches to joint surface repair: a research perspective. Osteoarthritis Cartilage. 2013;21(7): 892-900.

14. Foldager CB. Advances in autologous chondrocyte implantation and related techniques for cartilage repair. Dan Med J. 2013;60(4):B4600.

15. Goyal D, Goyal A, Keyhani S, Lee EH, Hui JH. Evidence-based status of second- and third-generation autologous chondrocyte implantation over first generation: a systematic review of level I and II studies. Arthroscopy. 2013;29(11):1872-1878.

16. Demoor M, Ollitrault D, Gomez-Leduc T, et al. Cartilage tissue engineering: molecular control of chondrocyte differentiation for proper cartilage matrix reconstruction. Biochim Biophys Acta. 2014;1840(8): 2414-2440.

17. Niemeyer P, Porichis S, Steinwachs M, et al. Long-term outcomes after first-generation autologous chondrocyte implantation for cartilage defects of the knee. Am J Sports Med. 2014;42(1):150-157.

18. Martinčič D, Radosavljevič D, Drobnič M. Ten-year clinical and radiographic outcomes after autologous chondrocyte implantation of femoral condyles. Knee Surg Sports Traumatol Arthrosc. 2014;22(6): 1277-1283.

19. Gillogly SD, Arnold RM. Autologous chondrocyte implantation and anteromedialization for isolated patellar articular cartilage lesions: 5- to 11-year follow-up. Am J Sports Med. 2014;42(4):912-920.

20. Gomoll AH, Gillogly SD, Cole BJ, et al. Autologous chondrocyte implantation in the patella: a multicenter experience. Am J Sports Med. 2014;42(5):1074-1081.

21. Meyerkort D, Ebert JR, Ackland TR, et al. Matrix-induced autologous chondrocyte implantation (MACI) for chondral defects in the patellofemoral joint. Knee Surg Sports Traumatol Arthrosc. 2014;22(10): 2522-2530. 
22. Nawaz SZ, Bentley G, Briggs TW, et al. Autologous chondrocyte implantation in the knee: mid-term to long-term results. J Bone Joint Surg Am. 2014;96(10):824-830.

23. Biant LC, Bentley G, Vijayan S, Skinner JA, Carrington RW. Long-term results of autologous chondrocyte implantation in the knee for chronic chrondral and osteochondral defects. Am J Sports Med. 2014;42(9): 2178-2183.

24. Kwak SK, Kern BS, Ferkel RD, Chan KW, Kasraeian S, Applegate GR. Autologous chondrocyte implantation of the ankle: 2- to 10-year results. Am J Sports Med. 2014;42(9):2156-2164.

25. Behery OA, Harris JD, Karnes JM, Siston RA, Flanigan DC. Factors influencing the outcome of autologous chondrocyte implantation: a systematic review. J Knee Surg. 2013;26(3):203-211.

26. Enea D, Cecconi S, Busilacchi A, Manzotti S, Gesuita R, Gigante A. Matrix-induced autologous chondrocyte implantation (MACI) in the knee. Knee Surg Sports Traumatol Arthrosc. 2012;20(5):862-869.

27. Edwards PK, Ackland T, Ebert JR. Clinical rehabilitation guidelines for matrix-induced autologous chondrocyte implantation on the tibiofemoral joint. J Orthop Sports Phys Ther. 2014;44(2):102-119.

28. Outerbridge RE. The etiology of chondromalacia patellae. J Bone Joint Surg Br. 1961;43-B:752-757.

29. Ventura A, Memeo A, Borgo E, Terzaghi C, Legnani C, Albisetti W. Repair of osteochondral lesions in the knee by chondrocyte implantation using the MACI ${ }^{\circledR}$ technique. Knee Surg Sports Traumatol Arthrosc. 2012;20(1):121-126.

30. Roos EM, Roos HP, Lohmander LS, Ekdahl C, Beynnon BD. Knee Injury and Osteoarthritis Outcome Score (KOOS) - development of a self-administered outcome measure. J Orthop Sports Phys Ther. 1998;28(2):88-96.

31. Kessler S, Lang S, Puhl W, Stove J. [The Knee Injury and Osteoarthritis Outcome Score - a multifunctional questionnaire to measure outcome in knee arthroplasty]. Z Orthop Ihre Grenzgeb. 2003;141(3):277-282. German.

32. Ebert JR, Robertson WB, Woodhouse J, et al. Clinical and magnetic resonance imaging-based outcomes to 5 years after matrix-induced autologous chondrocyte implantation to address articular cartilage defects in the knee. Am J Sports Med. 2011;39(4):753-763.

33. Ebert JR, Fallon M, Zheng MH, Wood DJ, Ackland TR. A randomized trial comparing accelerated and traditional approaches to postoperative weightbearing rehabilitation after matrix-induced autologous chondrocyte implantation: findings at 5 years. Am J Sports Med. 2012;40(7):1527-1537.

34. Ebert JR, Munsie C, Joss B. Guidelines for the early restoration of active knee flexion after total knee arthroplasty: implications for rehabilitation and early intervention. Arch Phys Med Rehabil. 2014;95(6): 1135-1140.

35. Bauer S, Khan RJ, Ebert JR, et al. Knee joint preservation with combined neutralising high tibial osteotomy (HTO) and matrix-induced autologous chondrocyte implantation (MACI) in younger patients with medial knee osteoarthritis: a case series with prospective clinical and MRI follow-up over 5 years. Knee. 2012;19(4):431-439.
36. Genovese E, Ronga M, Angeretti MG, et al. Matrix-induced autologous chondrocyte implantation of the knee: mid-term and long-term follow-up by MR arthrography. Skeletal Radiol. 2011;40(1):47-56.

37. Saris DB, Vanlauwe J, Victor J, et al; TIG/ACT/01/2000\&EXT Study Group. Treatment of symptomatic cartilage defects of the knee: characterized chondrocyte implantation results in better clinical outcome at 36 months in a randomized trial compared to microfracture. Am J Sports Med. 2009;37 Suppl 1:10S-19S.

38. Ebert JR, Fallon M, Robertson WB, et al. Radiological assessment of accelerated versus traditional approaches to postoperative rehabilitation following matrix-induced autologous chondrocyte implantation. Cartilage. 2011;2(1):60-72.

39. Dunkin BS, Lattermann C. New and emerging techniques in cartilage repair: MACI. Oper Tech Sports Med. 2013;21(2):100-107.

40. Albrecht C, Tichy B, Zak L, Aldrian S, Nurnberger S, Marlovits S. Influence of cell differentiation and IL-1b expression on clinical outcomes after matrix-associated chondrocyte transplantation. Am J Sports Med. 2014;42(1):59-69.

41. Ebert JR, Edwards PK. The evolution of progressive postoperative weight bearing following autologous chondrocyte implantation in the tibiofemoral joint. J Sport Rehabil. 2014;23(3):192-202.

42. Ebert JR, Smith A, Fallon M, Wood DJ, Ackland TR. Correlation between clinical and radiological outcomes after matrix-induced autologous chondrocyte implantation in the femoral condyles. Am J Sports Med. 2014;42(8):1857-1864.

43. Messier SP, Gutekunst DJ, Davis C, DeVita P. Weight loss reduces knee-joint loads in overweight and obese older adults with knee osteoarthritis. Arthritis Rheum. 2005;52(7):2026-2032.

44. Ebert JR, Smith A, Edwards PK, Hambly K, Wood DJ, Ackland TR. Factors predictive of outcome 5 years after matrix-induced autologous chondrocyte implantation in the tibiofemoral joint. Am J Sports Med. 2013;41(6):1245-1254.

45. Vasiliadis HS, Wasiak J. Autologous chondrocyte implantation for full thickness articular cartilage defects of the knee. Cochrane Database Syst Rev. 2010;(10):CD003323.

46. Kon E, Filardo G, Condello V, et al. Second-generation autologous chondrocyte implantation: results in patients older than 40 years. Am J Sports Med. 2011;39(8):1668-1675.

47. Blackman AJ, Smith MV, Flanigan DC, Matava MJ, Wright RW, Brophy RH. Correlation between magnetic resonance imaging and clinical outcomes after cartilage repair surgery in the knee: a systematic review and meta-analysis. Am J Sports Med. 2013;41(6):1426-1434.

48. de Windt TS, Welsch GH, Brittberg M, et al. Is magnetic resonance imaging reliable in predicting clinical outcome after articular cartilage repair of the knee? A systematic review and meta-analysis. Am J Sports Med. 2013;41(7):1695-1702.

49. De Coninck T, Huysse W, Willemot L, Verdonk R, Verstraete K, Verdonk P. Two-year follow-up study on clinical and radiological outcomes of polyurethane meniscal scaffolds. Am J Sports Med. 2013;41(1): 64-72.
Drug Design, Development and Therapy

\section{Publish your work in this journal}

Drug Design, Development and Therapy is an international, peerreviewed open-access journal that spans the spectrum of drug design and development through to clinical applications. Clinical outcomes, patient safety, and programs for the development and effective, safe, and sustained use of medicines are a feature of the journal, which
Dovepress

has also been accepted for indexing on PubMed Central. The manuscript management system is completely online and includes a very quick and fair peer-review system, which is all easy to use. Visit http://www.dovepress.com/testimonials.php to read real quotes from published authors. 